\title{
Adjuvant therapy for intrahepatic carcinoma after surgical resection: chemotherapy and future perspectives
}

\author{
Wei Zhang, Tianqiang Song \\ Department of Hepatobiliary Cancer, Research Center for Prevention and Treatment of Liver Cancer, Tianjin Medical University Cancer Institute \\ and Hospital, National Clinical Research Center for Cancer, Key Laboratory of Cancer Prevention and Therapy, Tianjin's Clinical Research Center \\ for Cancer, Tianjin, China \\ Correspondence to: Dr. Wei Zhang; Dr. Tianqiang Song, MD, PhD. Department of Hepatobiliary Cancer, Tianjin Medical University Cancer Institute \\ and Hospital, National Clinical Research Center for Cancer, Key Laboratory of Cancer Prevention and Therapy, Tianjin's Clinical Research Center \\ for Cancer, Huan Hu Xi Road, Tianjin, 300060, China. Email: zhangweitjch@163.com; tjchi@hotmail.com. \\ Comment on: Altman AM, Kizy S, Marmor S, et al. Adjuvant chemotherapy for intrahepatic cholangiocarcinoma: approaching clinical practice \\ consensus? Hepatobiliary Surg Nutr 2020;9:577-586.
}

Submitted Sep 08, 2021. Accepted for publication Oct 18, 2021.

doi: $10.21037 / \mathrm{hbsn}-21-368$

View this article at: https://dx.doi.org/10.21037/hbsn-21-368

Intrahepatic cholangiocarcinoma is a highly aggressive and malignant liver cancer. The main reason for its poor prognosis is the low opportunity of surgery and the high rates of postoperative recurrence and metastasis. Thus, reducing postoperative recurrence and metastasis rate is the key to improving prognosis. Although some retrospective studies have attested to the value of adjuvant therapy in patients with $\mathrm{R} 1$ resection or N1/T3/T4 intrahepatic cholangiocarcinoma (1), no recommended standard yet exists in intrahepatic cholangiocarcinoma primarily because the only 2 completed phase III clinical trials, the PRODIGE 12-ACCORD 18 trial (2) and the BILCAP trial (3), failed to achieve survival benefit in the chemotherapy arm in the adjuvant setting. However, one of the possible reasons for the negative results of the 2 studies is that patients with R1/ N1/T3/T4 were not differentiated. One randomized phase III trials (JCOG1202, ASCOT) of adjuvant S-1 therapy $v$ s. observation alone in resected biliary tract cancer (4) and one trial (ACTICCA-1 trial) of adjuvant chemotherapy with gemcitabine and cisplatin compared to observation after resection of cholangiocarcinoma and muscle invasive gallbladder carcinoma (5) are ongoing and awaiting future results.

In the study published by Altman and colleagues (6), postoperative adjuvant chemotherapy was confirmed to be effective in patients with $\mathrm{N} 1$ or $\mathrm{T} 3 / \mathrm{T} 4$ intrahepatic cholangiocarcinoma although the evidence level was not high because of missing data and insufficient information on the regimen and duration of the postoperative adjuvant chemotherapy. The data of the study were from the Surveillance Epidemiology and End Results (SEER) program database, representing the largest cohort of its kind thus far, and the study contributed evidence towards reaching a consensus in clinical practice.

The key to successful postoperative adjuvant therapy lies in identifying patients with residual cancer or microresidual cancer and providing effective treatment to these patients. Therefore, patients who need postoperative adjuvant therapy should first be identified. In Altman's study, among lymph node-negative patients, the median survival time of patients receiving chemotherapy was 46 months, while the median survival time of patients not receiving chemotherapy was 59 months $(\mathrm{P}=0.08)$, which suggests that adjuvant chemotherapy may not only be futile but even harmful to those patients who do not need it. A newly published study evaluating adjuvant transcatheter arterial chemoembolization (TACE) for TNM stage I intrahepatic cholangiocarcinoma also showed the harmful effect of adjuvant TACE for patients with early-stage tumors (7). Second, it is necessary to determine how to provide effective treatment to patients with residual cancer or minimal residual cancer. Early studies have shown that in patients with advanced biliary tract cancer including intrahepatic cholangiocarcinoma, the objective response rate (ORR) of 
chemotherapy is still low, ranging from 15\% (single-agent gemcitabine) (8) to $20.5 \%$ [gemcitabine hydrochloride plus oxaliplatin (GemOX)] (8) to $29.8 \%$ (gemcitabine plus S-1) to $30 \%$ (gemcitabine plus nab-paclitaxel) (9). Ultimately, the tumor will become resistant to chemotherapy, with progression-free survival (PFS) ranging from 3.4 months to 8 months (10). In the adjuvant setting, postoperative chemotherapy should be effective with low toxicity and a reduced tendency to induce resistance. Whether there exists options superior to chemotherapy remains unclear.

Recently, precision medicine has provided an opportunity for improving systemic treatment of cholangiocarcinoma (11). For example, patients with microresidual cancer can be identified by liquid biopsy of circulating tumor (ctDNA) or cell-free (cfDNA) and other technologies (12) in addition to pathological parameters such as $\mathrm{R} 1 / \mathrm{N} 1 / \mathrm{T} 3 /$ $\mathrm{T} 4$. With the progress of next-generation sequencing and the development of targeted therapy and immunotherapy, patients with certain targets can be precisely treated, as nearly half of cholangiocarcinoma cases have been shown to have driver genes (13). For example, one patient with the ATM-inactivating mutation, a homologous recombination deficiency (HRD), was successfully treated by olaparib and achieved an 18-month PFS (14). Other reports indicate that BRAF V600E can be targeted by a combination of trametinib and ceritinib, that FGFR2 mutation may be an ideal target for pemigatinib, and that EGFR mutation can be inhibited by afatinib. We have treated patients with IDH1 mutation by dasatinib, obtaining a PFS of 2 years (15). In another study, a patient with ERBB2positive lymph node metastasis and high tumor mutational burden (TMB) was given chemotherapy combined with immunotherapy and achieved complete tumor remission; the patient was still healthy and alive 3 years after operation without evidence of recurrence (11). In addition, many clinical cases with major partial response and even curative outcomes have yet not been published. Single or combined immunotherapy (with radiotherapy, chemotherapy, or targeted therapy) can be given according to certain markers of immunotherapy including microsatellite instability (MSI)-high, TMB-high, or programmed death-ligand 1 (PD-L1)-high status. Indeed, we can be cautiously optimistic that targeted therapy and immunotherapy will prove to be beneficial in the adjuvant setting. As a consequence, the position of chemotherapy may decline as combined targeted and immunotherapy gains prominence in the future.

\section{Acknowledgments}

Funding: This research was supported by funds as follows: (I) National Natural Science Foundation of China, No. 81572434; (II) Ministry of Science and Technology, National Science and Technology Major Special Project: Prevention and Treatment of Major Infectious Diseases such as AIDS and Viral Hepatitis, 2018ZX10723204007-001; (III) "Young Medical Elites," Tianjin Health Commission, 2017-1-35; (IV) "Young Innovative Talents", Tianjin Medical University Cancer Institute and Hospital, 2018-2-8.

\section{Footnote}

Provenance and Peer Review: This article was commissioned by the editorial office, Hepatobiliary Surgery and Nutrition. The article did not undergo external peer review.

Conflicts of Interest: Both authors have completed the ICMJE uniform disclosure form (available at https://hbsn. amegroups.com/article/view/10.21037/hbsn-21-368/coif). The authors have no conflicts of interest to declare.

Ethical Statement: The authors are accountable for all aspects of the work in ensuring that questions related to the accuracy or integrity of any part of the work are appropriately investigated and resolved.

Open Access Statement: This is an Open Access article distributed in accordance with the Creative Commons Attribution-NonCommercial-NoDerivs 4.0 International License (CC BY-NC-ND 4.0), which permits the noncommercial replication and distribution of the article with the strict proviso that no changes or edits are made and the original work is properly cited (including links to both the formal publication through the relevant DOI and the license). See: https://creativecommons.org/licenses/by-nc-nd/4.0/.

\section{References}

1. Ma KW, Cheung TT, Leung B, et al. Adjuvant chemotherapy improves oncological outcomes of resectable intrahepatic cholangiocarcinoma: A metaanalysis. Medicine (Baltimore) 2019;98:e14013.

2. Edeline J, Benabdelghani M, Bertaut A, et al. Gemcitabine and Oxaliplatin Chemotherapy or Surveillance in 
Resected Biliary Tract Cancer (PRODIGE 12-ACCORD 18-UNICANCER GI): A Randomized Phase III Study. J Clin Oncol 2019;37:658-67.

3. Primrose JN, Fox RP, Palmer DH, et al. Capecitabine compared with observation in resected biliary tract cancer (BILCAP): a randomised, controlled, multicentre, phase 3 study. Lancet Oncol 2019;20:663-73.

4. Nakachi K, Konishi M, Ikeda M, et al. A randomized Phase III trial of adjuvant S-1 therapy vs. observation alone in resected biliary tract cancer: Japan Clinical Oncology Group Study (JCOG1202, ASCOT). Jpn J Clin Oncol 2018;48:392-5.

5. Stein A, Arnold D, Bridgewater J, et al. Adjuvant chemotherapy with gemcitabine and cisplatin compared to observation after curative intent resection of cholangiocarcinoma and muscle invasive gallbladder carcinoma (ACTICCA-1 trial) - a randomized, multidisciplinary, multinational phase III trial. BMC Cancer 2015;15:564.

6. Altman AM, Kizy S, Marmor S, et al. Adjuvant chemotherapy for intrahepatic cholangiocarcinoma: approaching clinical practice consensus? Hepatobiliary Surg Nutr 2020;9:577-86.

7. Liu G, Guo W, Wang H, et al. Influence of postoperative adjuvant transarterial chemoembolization on the prognosis of early-stage intrahepatic cholangiocarcinoma: a single center study. Ann Palliat Med 2021;10:3673-83.

8. André T, Reyes-Vidal JM, Fartoux L, et al. Gemcitabine and oxaliplatin in advanced biliary tract carcinoma: a phase
II study. Br J Cancer 2008;99:862-7.

9. Sahai V, Catalano PJ, Zalupski MM, et al. Nab-Paclitaxel and Gemcitabine as First-line Treatment of Advanced or Metastatic Cholangiocarcinoma: A Phase 2 Clinical Trial. JAMA Oncol 2018;4:1707-12.

10. Zhang W, Zhou H, Wang Y, et al. Systemic treatment of advanced or recurrent biliary tract cancer. Biosci Trends 2020;14:328-41.

11. Zhang W, Shi J, Wang Y, et al. Next-generation sequencing-guided molecular-targeted therapy and immunotherapy for biliary tract cancers. Cancer Immunol Immunother 2021;70:1001-14.

12. Rizzo A, Ricci AD, Tavolari S, et al. Circulating Tumor DNA in Biliary Tract Cancer: Current Evidence and Future Perspectives. Cancer Genomics Proteomics 2020;17:441-52.

13. Lamarca A, Barriuso J, McNamara MG, et al. Molecular targeted therapies: Ready for "prime time" in biliary tract cancer. J Hepatol 2020;73:170-85.

14. Zhang W, Shi J, Li R, et al. Effectiveness of Olaparib Treatment in a Patient with Gallbladder Cancer with an ATM-Inactivating Mutation. Oncologist 2020;25:375-9.

15. Ma B, Meng H, Tian Y, et al. Distinct clinical and prognostic implication of IDH1/2 mutation and other most frequent mutations in large duct and small duct subtypes of intrahepatic cholangiocarcinoma. BMC Cancer 2020;20:318.
Cite this article as: Zhang $W$, Song T. Adjuvant therapy for intrahepatic carcinoma after surgical resection: chemotherapy and future perspectives. HepatoBiliary Surg Nutr 2021;10(6):878-880. doi: 10.21037/hbsn-21-368 